\title{
Incidental diagnosis of mesh plug migration
}

A 59-year old patient was referred in April 2012 for a routine colonoscopy 3 years after undergoing polyp ablation. His past medical history was significant for periumbilical mesh herniorrhaphy in 2010. The patient was asymptomatic at admission. The physical examination and laboratory findings were normal. During colonoscopy, a mass-like compression of the cecum with a mesh pattern of colonic mucosa was observed ( $\boldsymbol{~ F i g . 1}$ ). Incomplete migration of surgical mesh embedded in the colonic mucosa was diagnosed. There was no ulceration of mucosa, no protrusion of mesh into the colonic lumen, and no inflammation. Because the patient was asymptomatic, conservative management was decided. The patient was informed of the possible outcomes of complete migration and bowel perforation.

The use of mesh has become the gold standard for hernia repair surgery. Migration of mesh is a complication of this procedure. Primary migration occurs when an inadequately secured mesh migrates along an adjoining path of least resistance or when a relatively secure mesh is displaced by external forces. Secondary migration leads to erosion of surrounding tissue, with slow and gradual movement of the mesh through the transanatomic planes probably due to foreign body reaction with the mesh. Migrations have been described mainly after surgery for inguinal or umbilical hernia of the urinary bladder but also the gut [1]. Most of these cases were symptomatic with recurrent abdominal pain or occlusion. However, some cases of persistent abdominal pain or irritable bowel syndrome-like symptoms due to migration of a mesh have been described $[1,2]$. Consequently, due to the increasing number of case series of mesh surgery being reported, it has been suggested that incidence of this complication will increase in the future [3].

Mesh migration should be kept in mind as a potential cause of abdominal symptoms
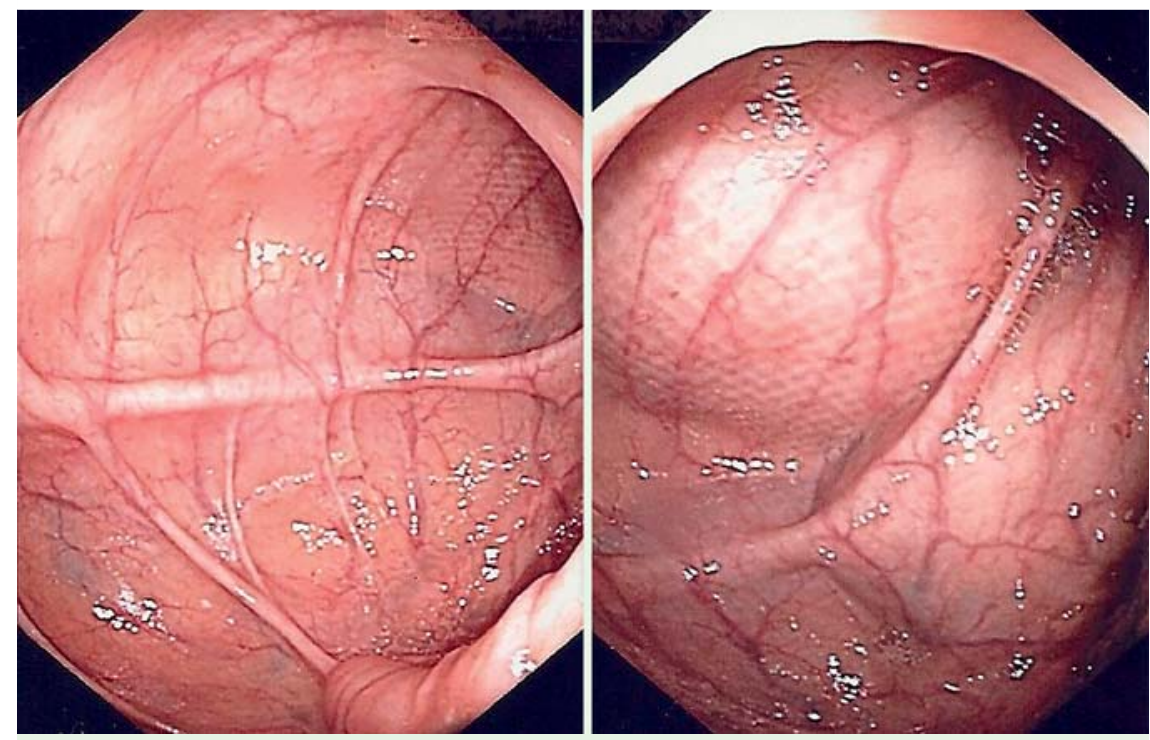

Fig. 1 Endoscopic view of mesh embedded in the cecal mucosa.

in these patients. To our knowledge, this is the first case of incidental discovery of mesh migration in an asymptomatic patient.

Endoscopy_UCTN_Code_CCL_1AD_2AI

Competing interests: None

\section{A. Garioud, X. Amiot}

Service d'hépato-gastroentérologie, Hôpital Tenon - AP-HP, Paris, France

\section{References}

1 Daas AY, Matthew B, Elijah D et al. An unexpected mass: mesh migration into the cecum following inguinal hernia repair. Gastroenterol Hepatol 2009; 5: 361 - 363

2 El Hakam MZ, Sharara I, Chedid V. Persistent left lower abdominal pain. Gastroenterology 2010; 138: e5-e6

3 Hamouda A, Kennedy J, Grant $N$ et al. Mesh erosion into the urinary bladder following laparoscopic inguinal repair; is this the tip of the iceberg? Hernia 2010; 14: 317-319
Bibliography

DOI http://dx.doi.org/

10.1055/s-0032-1326256

Endoscopy 2013; 45: E119

(c) Georg Thieme Verlag KG

Stuttgart · New York

ISSN 0013-726X

Corresponding author

\section{A. Garioud, MD}

Service d'hépato-gastroentérologie

Hôpital Tenon - AP-HP

4 , rue de la Chine

Paris 75020

France

Fax: +33-1-56017009

armand.garioud@tnn.aphp.fr 